\title{
The design and implementation of one wireless pulse monitor and alarm system
}

\author{
Chenghu Zhang \\ Computer Department, Chengdu Medical College, Chengdu, 610083, China \\ Email: zhang.cheng.hu@163.com
}

Keywords: Single Chip Microcomputer; The pulse sensor; Wireless Communication

\begin{abstract}
In this article, we introduce one kind of pulse monitoring system which based on IOT(Internet of things) and used for monitoring the patients' condition. First of all, the pulse sensor gather the pulse information of a patient and send the information to the pulse signal transceiver. Then the pulse signal transceiver send the signal to the main control device, and then the main control device judge the information and send the results to the user through GSM text messages module at last.
\end{abstract}

\section{Preface}

Body temperature, pulse, blood pressure, breath is main sign of life. The changes of pulse can indirectly reflect the changes of heart rate, blood pressure, respiration, body temperature and other signs.[1]So developing one sign monitor and alarm system which mainly measure the pulse signal has practical significance.

\section{The overall design of the system}

One hospital has many wards (here, we set the ward's number as $\mathrm{n}$, and the serial number is R1 $\mathrm{Rn}$ ), and every ward has one pulse's signal transceiver. One ward has several hospital beds (here, we set the bed's number as $\mathrm{m}$, and the serial number is R1-1 Rn-m), every bed has one pulse's signal pick device( the serial number is Pr1-1 Prn-m). The main control device receive the packaged signal, deal and judge it, and save the normal signal, if the signal is abnormal, the main control device will activate the GSM module and send message to the correspond doctors and nurses to alarm. The system block diagram is just as blow:

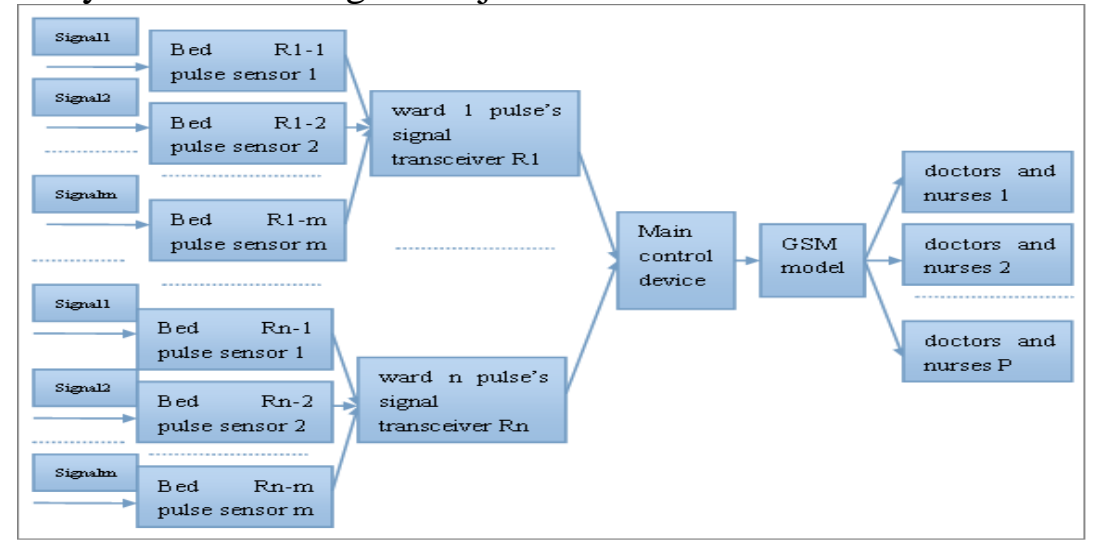

Picture 1 system block diagram

\section{Hardware design}

Signal pick device module. It include HKG-07A infrared ray pulse sensor, Atmel mega 328 SCM (Single Chip Microcomputer) and NEC (Nippon electronic company) infrared ray generator. The HKG-07A can detect the changes of the volume of microvascular within the fingers which is caused by the beating of heart, and pick the analog signal; Then amplify the signal, adjust, and deprive the high-frequency noise of the signal, through the A/D (analog/digital) translation circuit to 
get the correspond electric pulse signal. At last, synchronously output the digital voltage signal[2]. The signal dirrectly connect with the I/O interface of the SCM and transfer the pulse signal.

Atmel mega 328 SCM and NEC infrared ray generator. We use Atmel mega 328 SCM to control the pulse sensor pick the pulse signal of the patient and transfer the signal to digital signal, and through the NEC infrared ray generator to send the digital signal to the hospital pulse signal transceiver.

Pulse signal transceiver. Pulse signal transceiver is composed of Atmel Mega 328 SCM, NEC standard infrared receiving tube. The Atmel Mega 328 SCM control the infrared receiving tube to receive the infrared signal which come from the pulse signal pick device and pack the signal and then send to the main control device.

The main control module. The main control module is composed of Atmel mega 2560 SCM and infrared receiving tube. Atmel mega 2560 SCM is a 32 bit micro controller which can deal and control data. It has Avr as the core, at the same time has 64 or 128byte Flash memory, 256 byte inner RAM and 716 bit timer and 2 calculator, 9 programmable IO interface, a full duplex serial communication port, on-chip oscillator and clock circuit [3].

GSM text messages module. GSM text messages module is composed of Atmel GSM GPRS SHEILD expansion board and EFCOM wireless transmission device. When abnormal situation appear, the main control module send "AT" through serial port to GSM text messages module, ask GSM text messages module to send the alarm text messages to the doctors and nurses[4].

\section{Software design}

The software mainly realizes pulse digital signal receiving, storage, intelligent judgment and promoting GSM text messages module alarm. The program flow chart just like below:

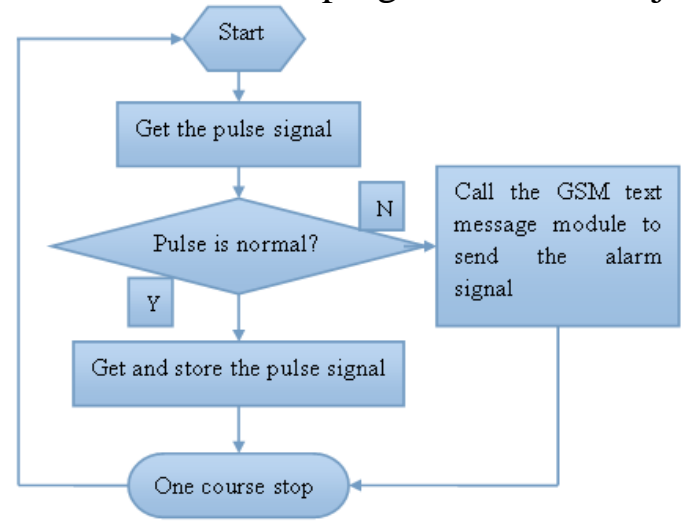

Picture 2 The main program flow chart

Signal pick program design. The signal pick module mainly finish the patient's pulse signal picking and dealing, and sending the digital signal of the pulse message through the I/O port to the infrared generator and to the correspond pulse signal transmitting and receiving device[5]. We use the clock function of the SCM to record the time of the appearance of low voltage and the high voltage, we take them as $\mathrm{t} 1$ and $\mathrm{t} 2$ respectively, and get and save the time $\mathrm{T} 1(\mathrm{~T} 1=\mathrm{t} 1+\mathrm{t} 2)$ which indicate the time that get one char, and do circle 15 times, and get every $\mathrm{T} 2$, and then get the average of the T(the $1 \mathrm{~T} 1$ add $14 \mathrm{~T} 2$, and use sum to divide the 15). So, we can use 1 to divide the $\mathrm{T}$ to get the estimated value of the pulse frequency. The part code just like as below:

\#include $<$ IRremote.h> //Quote the IRromote function library

\#define a A5 // a as port A5

Int $\mathrm{p}=0$; $\quad$ // pulse frequency $\mathrm{p}$

Int $\mathrm{i}=0$;

Int $\mathrm{T}=0$; $\quad$ // the average interval time of two pulse $\mathrm{T}$

Unsigned long t1; //high voltage time t1

Unsigned long t2 //low voltage time t2

Unsigned long T1; I/The first overall convert time T1

Unsigned long T2; $\quad$ //one overall convert time T2

Void setup() 
\{

pinMode(a,INPUT);

serial.begin(9600);

t1=pulseIn(a,HIGH,6000000); //high voltage

$\mathrm{t} 2=$ pulseIn(a,LOW,6000000); //low voltage

$\mathrm{T} 2=\mathrm{t} 1+\mathrm{t} 2$;

// one overall convert time

$\mathrm{T} 2=$ double $(\mathrm{T} 2)$;

$\mathrm{T} 2=60 / \mathrm{T} 2$;

PinMode(3,OUTPUT);

digitalWrite(3,HIGH); //light the LED test(need exam through mobile telephone

delay(3000);

//wait 3 seconds

\}

digitalWrite(3, LOW);

Void loop()

\{

For $(\mathrm{i}=1 ; \mathrm{i}<=15 ; \mathrm{i}++)$

\{

t1=pulseIn(a, HIGH, 6000000);

$\mathrm{t} 2=$ pulseIn(a, LOW, 6000000);

$\mathrm{T} 1=\mathrm{t} 1+\mathrm{t} 2$;

$\mathrm{T} 1=$ double(T1);

$\mathrm{T} 1=\mathrm{T} 1 / 1000000$;

$\mathrm{T} 1=60 / \mathrm{T} 1$;

$\mathrm{T}=(\mathrm{T} 1+\mathrm{T} 2) / 2$;

$\mathrm{T} 2=\mathrm{T} 1$;

Delay(1);

\}

\}

\{

Serial.printIn(T); //print serial port

Delay(5) ;

\}

The Wireless dispatching and receiving of the pulse signal. In this project, we define the leg of infrared receiver as A0; we convert analog signal to decimal digital signal which wrap output. In order to make the results more clearly observed, we add a blank line after each receiving code, and then receive the next data. Part of the code just like below:

\#include $<$ IRremote.h>

int RECV_PIN=A0; $\quad$ //define the leg of the infrared receiver as A0

IRrecy_irrecy(RECV_PIN);

Decode results;

Void setup()

\{

serial.begin( 9600);

Irrecv.enableIRIn(); //initiate the infrared receiver

\}

Void $\operatorname{loop}()$

\{

If (irrecy.decode( \&results)

\{

serial.printIn(results.value, DEC); // output decimal wrap signal

Serial.printIn();

Irrecv.resume();

//add a blank

\}

//receive the next data

\}

The intelligent judgment of the pulse signal. Taking into account the instrument error, in this project, we enlarge the pulse range to 50-110 times per minute, the interval time between every detection is one minute. When we run program, if the pulse number exceed the range, the main control module will call the GSM module to send the text message to alarm. For avoid the appearance of the wrong code, we output one judgment three times, if one time we get the right 
code, it indicate that the data is good, otherwise indicate that the data is wrong. Part of the code like as below:

\#define a A5 //define a as the port A5

If $(\mathrm{T}>=50 \& \& \mathrm{~T}<=110)$

\{

Serial.printIn(T);

$\mathrm{b}=\mathrm{T}$;

for (int $\mathrm{i}=0 ; \mathrm{i}<3 ; \mathrm{i}++)$

\{

Serial.print(“SendIR:”);

iresend.sendNEC(b,32);

\}

delay(40);

\section{Conclusion}

In this system, we use the wireless communication technology as a strong support, use the sophisticated hardware platform, and basically realize the real-time monitoring of patient's pulse signal, and the wireless transmission function. This system has the advantage of very strong realization, high safety and stability, simple and easy to operate, independent extensible, can help the nurses monitoring the patient's vital signs, and thereby reduce the workload of medical staff, and greatly avoid the huge damage to the patient due to the delay treatment, and effectively ease the doctor-patient relationship[6]. But we have not yet achieved the network among the multiple beds and many wards. With the support from our country to the IOT and increasing serious "aging" problems and the increasing incidence and mortality of cardiovascular diseases, the demand for wireless medical monitoring equipment increase rapidly. If the device can be modify smaller and more convenient, it will be welcomed by more old people, this kind of remote medical monitoring system will have broad market prospects.

\section{References}

[1] XinLi, Xing Li, Jingwei Dong. The vital signs monitoring system based on ZigBee[J]. Information technology, 2009,(8)

[2] Lanjun Liu, Peihong Mao. Design and implementation of a simple intelligent robot which can cut grass based on ArduinoMega2560 single chip microcomputer[J]. Journal of Anhui Agri.Sci.2012,40(36):17899-17901,17903.

[3] Quan Guo, XiangFang Zhu. Measurement and analysis of human pulse[J]. Shanghai Biomedical Engineering.2006,(2):34-35.

[4] YuTai Cai, ZeFeng Li, JiangWei Lin. The pulse monitor which based on the Bluetooth and the Smart phones[J]. Medical equipment, 2013,01:13-16.

[5] Qun Meng, LongPin Yang. The present status of development and Research on the key techniques about the the medical Internet of things. Chinese Journal of health information management, 2013,10(4)

[6] HaiQuan Xiang. On 2013 the medical information. EHC Round Table Forum 